\title{
Association between tooth loss and hypertension among older Chinese adults: a community-based study
}

Dongxin Da ${ }^{1,2+}$, Fei Wang ${ }^{3,4 \dagger}$, Hao Zhang ${ }^{1,2}$, Xiaoli Zeng ${ }^{1,2}$, Yiwei Jiang ${ }^{1,2}$, Qianhua Zhao ${ }^{4}$, Jianfeng Luo ${ }^{3}$, Ding Ding ${ }^{4}$, Ying Zhang ${ }^{1,2^{*}}$, Bei $W u^{5,6^{*}}$ and and the Shanghai Aging Study

\begin{abstract}
Background: The purpose of the study is to examine the association between tooth loss and hypertension among older community residents in urban China.

Methods: This study included 3677 participants aged $\geq 50$ years from the Shanghai Aging Study. We determined the number of teeth missing from questionnaires. Hypertension was defined as the mean of two measurements of systolic blood pressure (SBP) (140 mmHg or higher), diastolic blood pressure (DBP) (90 mmHg or higher) or physician-diagnosed hypertension confirmed from medical records. A multivariable logistic regression model was used to investigate the association between tooth loss and hypertension.

Results: The average number of missing teeth among study participants was 9.67. Among them, participants with hypertension had lost an average of 10.88 teeth, significantly higher than those without hypertension (8.95) $(p<0.0001)$. After adjusting for covariates (socio-demographic characteristics, health behaviors and other chronic conditions), teeth lost (15 or more) was significantly associated with grade III hypertension, with $\mathrm{OR}=1.55(95 \% \mathrm{Cl} 1.09-2.20)$.
\end{abstract}

Conclusions: Significant tooth loss maybe associated with severe hypertension among older Chinese adults. Prevention of tooth loss is important to the overall health of this population.

Keywords: Teeth, Hypertension, Community, Older adults

\section{Background}

Hypertension is considered the leading risk factor for life-threatening diseases [1-4].Approximately $54 \%$ of stroke and $47 \%$ of ischemic heart disease cases have increased blood pressure [5].According to a national survey of cardiovascular disease conducted in China in 2015, the prevalence of hypertension increased from 2002 (17.7\%) to 2012 (25.2\%). In 2010, hypertension caused approximately 2 million deaths in China [6].

The number of remaining teeth is an important indicator for oral health [7]. According to the 2005

\footnotetext{
* Correspondence: zhangyingcmu@vip.163.com; bei.wu@nyu.edu ${ }^{\dagger}$ Dongxin Da and Fei Wang contributed equally to this work.

'Department of Preventive Dentistry, Shanghai Stomatological Hospital,

Fudan University, 356East Beijing Rd, Shanghai 200001, China

${ }^{5}$ Rory Meyers College of Nursing and NYU Aging Incubator, New York

University, 433 First Avenue, New York, NY 10010, USA

Full list of author information is available at the end of the article
}

Chinese National Oral Health Survey, 4.5\% of elderly Chinese were edentulous and those aged 65-74 years had lost an average of 10 teeth [8]. Numerous studies have shown that periodontitis and tooth loss caused by oral inflammation is significantly associated with hypertension, excluding other risk factors [4]. Numerous studies on tooth loss and hypertension have been carried out around the world. These studies found a significant association between hypertension or increased blood pressure and periodontal disease or tooth loss [9-21].

Studies conducted in high income countries showed a great variation in study design, sample sources and data analyses in this topic area. No studies have been conducted among the Chinese population. Due to differences in health care system, culture, genetic factors and health literacy, it is unclear whether the evidence

(c) The Author(s). 2019 Open Access This article is distributed under the terms of the Creative Commons Attribution 4.0 International License (http://creativecommons.org/licenses/by/4.0/), which permits unrestricted use, distribution, and 
generated from other countries is generalizable to the Chinese population. For example, people in western countries consume more high-cholesterol foods than Chinese people. The prevalence of hypertension among Americans is $32 \%$ but is $25.2 \%$ in China [ 6 , 22].Therefore studies on older Chinese people are necessary. In addition, the prevalence of hypertension and cardiovascular disease is rapidly rising in China. Identifying risk factors for hypertension and cardiovascular disease has important clinical and policy implications for healthcare professionals and policy makers to develop targeted interventions and programs to prevent and treat hypertension. To address the knowledge gap in the literature, this study aimed to examine the association between tooth loss and hypertension among older community residents in urban Shanghai, China.

\section{Methods}

\section{Study participants}

All participants were recruited from the Shanghai Aging Study (SAS). The SAS aimed to investigate the prevalence of dementia and mild cognitive impairment among older adults living in Jingansi community, Shanghai [23]. Between January 2010 and December 2012, 3836 residents aged 50 years or older were enrolled by SAS, excluding any living in nursing homes or other institutions. Detailed inclusion and exclusion criteria were published elsewhere [23]. Finally, 3677 out of 3836 participants with complete and reasonable self-reported tooth loss were selected in the current study.

\section{Blood pressure measurement and definition of hypertension}

In a seated position, blood pressure was measured twice after at least 5 min of rest using a digital electronic tensiometer (M4; OMRON Corp., Kyoto, Japan); a proper cuff for the patient's left arm at heart level was used [24]. Hypertension was defined as the mean of two measurements of systolic blood pressure (SBP) $(140 \mathrm{mmHg}$ or higher), diastolic blood pressure (DBP) $(90 \mathrm{mmHg}$ or higher) or physician-diagnosed hypertension confirmed from their medical records $[1,25,26]$. According to the 2010 Chinese guidelines for the management of hypertension, stage III hypertension was defined as the mean of two measurements of SBP $(180 \mathrm{mmHg}$ or higher $)$ or DBP (110 $\mathrm{mmHg}$ or higher), stage II hypertension as the mean of two measurements of SBP $(160-179 \mathrm{mmHg})$ or DBP (100-109 $\mathrm{mmHg}$ ), stage I hypertension as the mean of two measurements of SBP $(140-159 \mathrm{mmHg})$ or DBP (90-99 $\mathrm{mmHg}$ ) and normal blood pressure as the mean of two measurements of SBP (less than $140 \mathrm{mmHg}$ ) or DBP (less than $90 \mathrm{mmHg}$ ) [26, 27].

\section{Observation of tooth loss}

Participants in the study received a self-administered oral health questionnaire (Additional file 1) and answered questions about their numbers of missing teeth by themselves. The interviewers counted the number of missing teeth (including third molars) and reviewed the questionnaires. After analyzing the data distribution, we categorized the participants into three groups based on tertiles of the data distribution of tooth loss:(1) $0-3$ missing; (2) 4-14 missing; and (3) $\geq 15$ missing.

\section{Data collection of other variables}

Trained research nurses and physicians interviewed the participants face-to-face in order to obtain all relevant information including demographic variables and participants' socioeconomic status. Demographic variables included age, sex, body mass index (BMI) and number of formal years of education. The BMI was calculated according to the following formula: person's weight in kilograms/height in meters squared. Obesity was defined as BMI $\geq 27.5 \mathrm{~kg} / \mathrm{m}^{3}$ based on WHO definition for Asian populations was defined as BMI $\geq 27.5 \mathrm{~kg} / \mathrm{m}^{3}$ based on WHO definition for Asian populations. Detailed definitions of variables mentioned above have been published elsewhere [23]. Finally, medical histories were collected by self-report and confirmed with participants' medical records, and consisted of diabetes and heart disease (including coronary artery disease and arrhythmia).

\section{Statistical analysis}

For the current data, rates of participants with $\geq 15$ tooth lost in hypertension group and non-hypertension group were 0.268 and 0.192 , due to the sample size of 3677 , the power of test $(1-\beta)$ exceeded $95 \%$ with $\alpha=0.05$, according to the below formulation. Therefore the sample size was sufficient in our study.

$$
n=\frac{Z_{\alpha / 2} \sqrt{2 \mathrm{p}(1-\mathrm{p})}+Z_{\beta} \sqrt{\mathrm{p}_{0}\left(1-\mathrm{p}_{0}\right)+\mathrm{p}_{1}\left(1-\mathrm{p}_{1}\right)}}{\left(\mathrm{p}_{1}-\mathrm{p}_{0}\right)^{2}}
$$

Continuous variables were described in mean and standard deviation (SD), and frequencies (\%) were used for categorical variables. We used the Student ttest, Pearson Chi-squared test, analysis of variance (ANOVA) and Cochran-Mantel-Haenszel Chisquared to compare the variables. The association between the number of teeth missing and hypertension was examined by three ordinal logistic regression models. Odds ratio (OR) and 95\% confidence intervals (CI) presented the measurement of the association. Model 1 was a univariate model and Models 2 and 3 were multivariable models. Model 2 was adjusted for age and sex; Model 3 was further adjusted for 
confounders such as age, sex, socioeconomic factors (education and household income), health compromising behaviours (smoking and drinking), Obesity and medical history (heart disease and diabetes). The group of people with $\leq 3$ teeth lost was the reference category for all models.

The $p$-values and 95\% CIs were estimated in a twotailed manner, and $p<0.05$ was considered significant. Data were analyzed using SAS 9.3 (SAS Institute Inc., Cary, NC, USA).

\section{Results}

The current study included 3677 community-dwelling older adults, aged60 years or above who responded the oral health questionnaire, and received blood pressure measurement. Among them, 1644(44.71\%) were men. The mean age was 70.23(SD 8.61) years and mean years of education was 11.69(SD 4.08) years. Among all the participants, 1339 were diagnosed with hypertension. Significant differences in age, BMI, cigarette smoking, heart disease, diabetes, dental caries, being obesity and tooth loss were found between participants with hypertension and non-hypertension. Participants with hypertension were older (mean 72.72,SD 8.62 years), had fewer years of education(mean 11.41,SD 4.49)and had higher prevalence of heart disease $(19.10 \%)$ and diabetes(19.24\%).Participants with hypertension had significantly more teeth missing (mean 10.88) than those without hypertension (mean 8.95) $(p<0.001)$ (Table 1$)$. More than one-quarter of participants with hypertension had lost $\geq 15$ teeth, which was higher compared to nonhypertension participants. Also, the number of teeth missing was associated with hypertension. Subjects with $\geq 15$ teeth lost reported higher prevalence of hypertension $(p<0.001)$ (Table 1$)$.

The ORs for number of teeth missing among participants with or without hypertension are shown in Table 2.The univariate Model 1 showed that participants who lost $\geq 15$ teeth had an OR for hypertension of $1.97(95 \%$ CI $1.65-$ $2.35)$, and OR for grade III hypertension of 3.44(95\% CI 2.52-4.69).After adjustment for sex and age, $\geq 15$ tooth lost was associated with a $21 \%(\mathrm{OR}=1.21,95 \%$ CI $1.00-1.47)$ higher odds of hypertension (Model 2). Model 3 showed that tooth loss of $\geq 15$ was significantly associated with grade III hypertension with OR $=1.55(95 \%$ CI 1.09-2.20).

\section{Discussion}

This cross-sectional study indicated a positive association between tooth loss and hypertension in community-dwelling older adults in Shanghai, China.

Table 1 Demographic, social economic and medical history of the participants with hypertension and non-hypertension

\begin{tabular}{|c|c|c|c|c|c|}
\hline Variates & ALL N = 3677 & Tooth loss $\leq 3 \mathrm{~N}_{1}=1574$ & Tooth loss $4-14 \mathrm{~N}_{2}=1296$ & Tooth loss $\geq 15 \mathrm{~N}_{3}=807$ & $P$ value \\
\hline \multicolumn{5}{|l|}{ Sex } & \multirow[t]{3}{*}{0.0002} \\
\hline Male, n(\%) & $1644(44.71)$ & $644(40.91)$ & $606(46.76)$ & $394(48.82)$ & \\
\hline Female, n(\%) & $2033(55.29)$ & $930(59.09)$ & $690(53.24)$ & $413(51.18)$ & \\
\hline Age,years, mean(SD) & $70.23 \pm 8.61$ & $67.51 \pm 7.99$ & $69.92 \pm 7.65$ & $76.06 \pm 8.41$ & $<.0001$ \\
\hline Education,years, mean(SD) & $11.69 \pm 4.08$ & $11.81 \pm 3.87$ & $11.89 \pm 3.86$ & $11.12 \pm 4.74$ & 0.0229 \\
\hline Body mass index, mean(SD) & $24.26 \pm 3.53$ & $24.14 \pm 3.33$ & $24.55 \pm 3.47$ & $24.03 \pm 3.96$ & 0.0002 \\
\hline \multicolumn{5}{|c|}{ Monthly household income, $n$ (\%) } & \multirow[t]{4}{*}{0.0039} \\
\hline$<500 \mathrm{RMB}$ & $37(1.01)$ & $11(0.71)$ & $13(1.01)$ & $13(1.62)$ & \\
\hline 500-1200RMB & $52(1.42)$ & $15(0.96)$ & $16(1.24)$ & $21(2.62)$ & \\
\hline$>1200 \mathrm{RMB}$ & $3564(97.56)$ & $1534(98.33)$ & $1262(97.75)$ & 768 (95.76) & \\
\hline Cigarette smoking, $n(\%)$ & $390(10.67)$ & $131(8.39)$ & $158(12.22)$ & $101(12.61)$ & 0.0006 \\
\hline Alcohol drinking, $n(\%)$ & $298(8.24 \%)$ & $122(7.87)$ & $103(7.98)$ & 73 (9.13) & 0.5434 \\
\hline Heart disease, $n(\%)$ & $443(12.13)$ & $142(9.13)$ & $146(111.28)$ & 155 (19.30) & $<.0001$ \\
\hline Diabetes & 498 (13.62) & $180(11.58)$ & $182(14.05)$ & $136(16.85)$ & 0.0016 \\
\hline $\mathrm{ADL}$, mean(SD) & $21.01 \pm 5.01$ & $20.71 \pm 4.68$ & $20.77 \pm 4.30$ & $21.97 \pm 6.40$ & $<.0001$ \\
\hline Obesity, n(\%) & $1435(38.87)$ & $579(36.79)$ & $552(42.59)$ & $304(37.67)$ & 0.0044 \\
\hline Hypertension & 1908 (51.89) & 749 (48.14) & 688 (53.09) & $471(58.36)$ & $<.0001$ \\
\hline \multicolumn{5}{|l|}{ Stage of hypertension } & \multirow[t]{5}{*}{$<.0001$} \\
\hline Non-hypertension & $1676(45.58)$ & $820(52.10)$ & $569(43.90)$ & $287(35.56)$ & \\
\hline । & 1179 (32.06) & $457(29.03)$ & $446(34.41)$ & $276(34.20)$ & \\
\hline$\|$ & $544(14.79)$ & $208(13.21)$ & 199 (15.35) & $137(16.98)$ & \\
\hline III & $278(7.56)$ & 89 (5.65) & 82 (6.33) & 107 (13.26) & \\
\hline
\end{tabular}




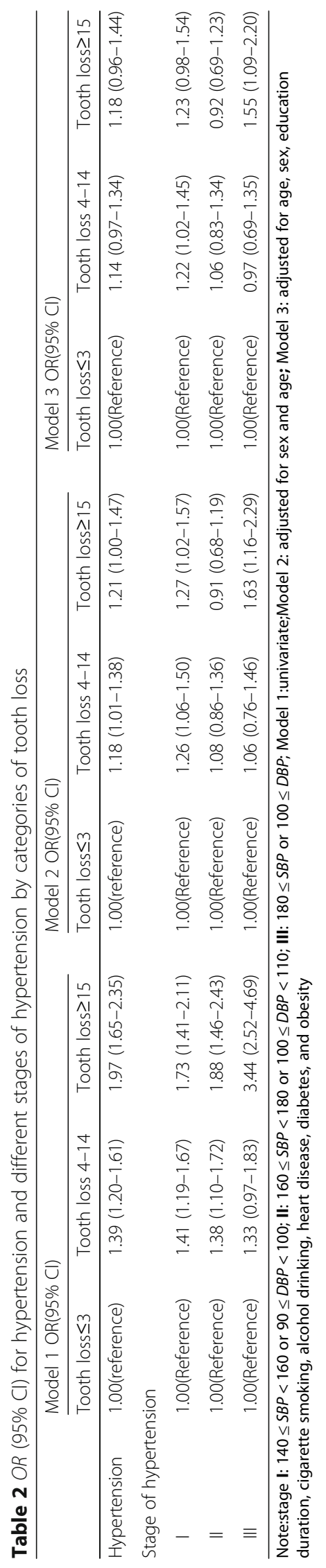


Loss of more than 15 teeth was positively associated with hypertension after adjusting for covariates.

Consistent with other observational studies, a strong association between tooth loss and hypertension was observed after controlling for a number of confounding variables. Some studies have reported that tooth loss is associated with higher SBP [14-16, 28, 29] and peripheral arterial disease among men [30].A crosssectional study in Indian adults indicated that participants with partial tooth loss had 1.62 times (95\% CI1.12-2.35) higher OR of developing hypertension after adjusting for all confounders, compared to those with no tooth loss [4].Moreover, the cross-sectional study of Peres et al. suggested that edentulous people have an $8.3 \mathrm{mmHg}(95 \% \mathrm{CI} 0.1-16.7)$ higher SBP compared to individuals with more than 10 teeth in both arches after adjustment [13].The significant association between missing teeth ( $>10$ missing) and hypertension was also observed among a subset of $<65$ years old in a French cohort study with $\mathrm{OR}=1.17$ (95\% CI 1.04-1.31) [31]. Ayo-Yusuf found in 2008 that total tooth loss was significantly associated with hypertension in South African adults, with $\mathrm{OR}=$ 1.35(95\% CI 1.02-1.78) [9].Additionally, after adjusting for obesity, hypercholesterolemia and hypertriglyceridemia, Taguchi et al. demonstrated that the OR of having hypertension in postmenopausal women with missing teeth was 3.59 (95\% CI 1.10-11.7) [15].

Several mechanisms have been proposed to explain the association between tooth loss and hypertension. Due to masticatory insufficiency, tooth loss may alter the eating habits of individuals and so cause less intake of vitamins, fiber and more cholesterol, consequently increasing risk of hypertension [11, 29, 32].Since China is traditionally an agricultural country, high-fiber food still constitutes most of the diet of the study participants. Subsequently, partial tooth loss might be the cause of reduced fiber intake, which is considered to be one risk factor for hypertension [4]. Other potential mechanisms include chronic periodontal inflammation caused by periodontal pathogens, which produce chronic systemic inflammation and vasculopathy and increase the risk of hypertension and cardiovascular diseases in those with periodontal disease [30, 33, 34]. This was further verified by a study which demonstrated a significant association between periodontal pockets and hypertension [17] but no association was found between hypertension and number of teeth [10].

Self-reported number of teeth has been proved effective and is widely used in observational studies. Previous studies conducted in the United States [34] and Japan [33] suggested no significant difference between selfreported number of teeth and the numbers from clinical examination data. In our study, the participants had an average of 9.67 missing teeth, comparable to the findings from large national oral health surveys using clinical examination data in China, in which adults aged 60-74 years had an average of 10 missing teeth [8]. Furthermore, to avoid recall bias, the interviewers observed, counted and confirmed the number of teeth missing for each participant [35], therefore we collected relatively accurate numbers for tooth loss in this study.

Tooth loss is an oral health problem accumulated across life span, and worsens in later life. Severe periodontal disease, unhealthy diet and poor dental care can lead to tooth loss. Given that tooth loss and hypertension are both common among older adults, it is possible that the association between hypertension and tooth loss is bidirectional among those with significant tooth loss [35](those who lost $\geq 15$ teeth in our study).

The strengths of our study are the relatively large sample size for a study of such scale. We adjusted for relevant confounders sequentially in our three models. However, the study has certain limitations. Firstly, hypertension is known to be a punctual measurement so that the absence of years of hypertension of an individual may be a disadvantage. Secondly, tooth loss may be associated with obesity and diabetes, which are independently associated with hypertension these variables are likely to be on the causal pathway between tooth loss and hypertension [36, 37]. Therefore, the adjustment in Model 3 might be inappropriate. In addition, although we adjusted for as many potential confounders as possible, residual and unmeasured confounding could not be completely ruled out. Thirdly, it's better to use inadequate dentition with individuals with fewer than 21 teeth to categorize tooth loss [38]. However, because of our relatively small sample size, there is significant difference in sample size between inadequate and adequate dentition. We will consider using the classification above in our future study with larger sample size. In the fourth place, as a study conducted in urban Shanghai, participants have a higher level of education than other areas in China. Therefore, the study findings may not be generalizable to the wider older population in China. Finally, our findings were based on a cross-sectional study, therefore we were only able to examine the association between tooth loss and hypertension.

\section{Conclusions}

In conclusion, among older Chinese adults, we observed an association between tooth loss and hypertension. Low numbers of remaining teeth might be considered as an impact factor for hypertension among the Chinese older population. Longitudinal and prospective studies are needed to further elucidate the causal association between oral health and hypertension. 


\section{Supplementary information}

Supplementary information accompanies this paper at https://doi.org/10. 1186/s12903-019-0966-3.

Additional file 1. Oral Health Assessment Questionaire for Adults

\section{Abbreviations}

ANOVA: Analysis of Variance; BMI: Body Mass Index; Cl: Confidence Interval; DBP: Diastolic Blood Pressure; OR: Odds Ratio; SAS: The Shanghai Aging Study; SBP: Systolic Blood Pressure; SD: Standard Deviation

\section{Acknowledgements}

Not applicable.

\section{Authors' contributions}

This work was conceptualized by $Y Z$ and BW and all approved the protocol. Data collection was done by DDa, HZ, XZ, YJ, QZ and DDi. Statistical analysis was undertaken by FW and JL. DDa, FW and DDi prepared the manuscript. YZ and BW are the guarantors of this paper. All authors have read and approved the manuscript, and ensure that this is the case.DDi should be contacted for the data.

\section{Funding}

The Clinical Research Plan of SHDC (16CR4018A) and Shanghai Stomatological Hospital School-level Research Project (SSDC-2016-06) supported analysis, interpretation of data and writing the manuscript. The Shanghai Brain-Intelligence Project from STCSM [grant number 16JC1420500], Scientific Research Project from STCSM [grant number 17411950701, 17411950106], Natural Science Foundation and Major Basic Research Program of Shanghai [grant number 16JC1420100], and National Natural Science Foundation of China [grant number 81773513] supported data collection of this study.

\section{Availability of data and materials}

The authors can share their relevant raw data supporting their findings. If any scientist wishes to use them for non-commercial purposes, without breaching participant confidentiality, he or she can contact the authors directly, and they will share their raw data freely with him or her.

\section{Ethics approval and consent to participate}

The Medical Ethics Committee of Huashan Hospital, Fudan University approved this study in Shanghai, China. We collected written informed consent from all the participants/their legal guardians.

\section{Consent for publication}

Not applicable.

\section{Competing interests}

The authors declare that they have no competing interests.

\section{Author details}

${ }^{1}$ Department of Preventive Dentistry, Shanghai Stomatological Hospital, Fudan University, 356East Beijing Rd, Shanghai 200001, China. ${ }^{2}$ Oral Biomedical Engineering Laboratory, Shanghai Stomatological Hospital, Fudan University, Shanghai, China. ${ }^{3}$ Department of Biostatistics, School of Public Health, Fudan University, Shanghai, China. ${ }^{4}$ Institute of Neurology, Huashan Hospital, Fudan University, Shanghai, China. ${ }^{5}$ Rory Meyers College of Nursing and NYU Aging Incubator, New York University, 433 First Avenue, New York, NY 10010, USA. ${ }^{6}$ NYU Aging Incubator, New York University, New York, NY, USA.

Received: 8 October 2018 Accepted: 21 November 2019

Published online: 09 December 2019

\section{References}

1. Kearney PM, Whelton M, Reynolds K, Muntner P, Whelton PK, He J. Global burden of hypertension: analysis of worldwide data. Lancet. 2005;365:21723.

2. Lim SS, Vos T, Flaxman AD, Danaei G, Shibuya K, Adair-Rohani H, et al. A comparative risk assessment of burden of disease and injury attributable to
67 risk factors and risk factor clusters in 21 regions, 1990-2010: a systematic analysis for the global burden of disease study 2010. Lancet. 2012;380:222460

3. Górski B, Nargiełło E, Grabowska E, Opolski G, Górska R. The Association Between Dental Status and Risk of Acute Myocardial Infarction Among Poles. Case-Control Study. 2016;25:861.

4. Singh A, Gupta A, Peres MA, Watt RG, Tsakos G, Mathur MR. Association between tooth loss and hypertension among a primarily rural middle aged and older Indian adult population. J Public Health Dent. 2016;76:198-205.

5. Lawes CM, Vander HS, Rodgers A. Global burden of blood-pressure-related disease, 2001. Lancet. 2008;371:1513-8.

6. Ma L, Wu Y, Wang W, Chen W. Interpretation of the report on cardiovascular diseases in China (2017). Chin J Cardiovasc Med. 2018:23(01):3-6.

7. Services HH, Washington. Healthy People 2010: Understanding and Improving Health. 2000;:73.

8. Qi X. Reports of the 3rd national oral health survey of China. People's Hygiene Press; 2008.

9. Ayo-Yusuf OA, Ayo-Yusuf IJ. Association of tooth loss with hypertension. S Afr Med J. 2008;98:381-5.

10. Holmlund A, Holm G, Lind L. Severity of periodontal disease and number of remaining teeth are related to the prevalence of myocardial infarction and hypertension in a study based on 4,254 subjects. J Periodontol. 2006;77: $1173-8$

11. Lowe G, Woodward M, Rumley A, Morrison C, Tunstall-Pedoe H, Stephen K. Total tooth loss and prevalent cardiovascular disease in men and women: possible roles of citrus fruit consumption, vitamin C, and inflammatory and thrombotic variables. J Clin Epidemiol. 2003;56:694-700.

12. Okoro CA, Balluz LS, Eke PI, Ajani UA, Strine TW, Town M, et al. Tooth loss and heart disease: findings from the behavioral risk factor surveillance system. Am J Prev Med. 2005:29(5 Suppl 1):50-6.

13. Peres MA, Tsakos G, Barbato PR, Silva DA, Peres KG. Tooth loss is associated with increased blood pressure in adults-a multidisciplinary populationbased study. J Clin Periodontol. 2012;39:824-33.

14. Rivas-Tumanyan S, Campos M, Zevallos JC, Joshipura KJ. Periodontal disease, hypertension, and blood pressure among older adults in Puerto Rico. J Periodontol. 2013:84:203-11.

15. Rivas-Tumanyan S, Spiegelman D, Curhan GC, Forman JP, Joshipura KJ. Periodontal disease and incidence of hypertension in the health professionals follow-up study. Am J Hypertens. 2012;25:770-6.

16. Taguchi A, Sanada M, Suei $Y$, Ohtsuka M, Lee $K$, Tanimoto $K$, et al. Tooth loss is associated with an increased risk of hypertension in postmenopausal women. Hypertension. 2004;43:1297-300.

17. Tsakos G, Sabbah W, Hingorani AD, Netuveli G, Donos N, Watt RG, et al. Is periodontal inflammation associated with raised blood pressure? Evidence from a national US survey. J Hypertens. 2010:28:2386-93.

18. Watt RG, Tsakos G, de Oliveira C, Hamer M. Tooth loss and cardiovascular disease mortality risk-results from the Scottish health survey. PLoS One. 2012;7:e30797.

19. Volzke H, Schwahn C, Dorr M, Aumann N, Felix SB, John U, et al. Inverse association between number of teeth and left ventricular mass in women. J Hypertens. 2007:25:2035-43.

20. Volzke H, Schwahn C, Hummel A, Wolff B, Kleine V, Robinson DM, et al. Tooth loss is independently associated with the risk of acquired aortic valve sclerosis. Am Heart J. 2005;150:1198-203.

21. Shin HS. Association between the number of teeth and hypertension in a study based on 13,561 participants. J Periodontol. 2018.

22. Greenland P, Peterson E. The new 2017 ACC/AHA guidelines "up the pressure" on diagnosis and treatment of hypertension. Jama. 2017;318:2083.

23. Ding D, Zhao Q, Guo Q, Meng H, Wang B, Yu P, et al. The Shanghai aging study: study design, baseline characteristics, and prevalence of dementia. Neuroepidemiology. 2014;43:114-22.

24. Alperovitch A, Blachier M, Soumare A, Ritchie K, Dartigues JF, RichardHarston S, et al. Blood pressure variability and risk of dementia in an elderly cohort, the Three-City study. Alzheimers Dement. 2014;10(5 Suppl):S330-7.

25. Gao XL, Yin GD, Bing YH, Jin YZ, Jin QH. Cardiovascular change induced by central hypertonic saline are accompanied by GABA release in awake rats. Zhongguo Ying Yong Sheng Li Xue Za Zhi. 2009;25:462-6.

26. Wang $\mathrm{P}$, Zeng $\mathrm{H}$. Research Progress on the relationship between hypertension and cognitive function. Chin Gen Pract. 2011;14:677-9.

27. Liu LS. 2010 Chinese guidelines for the management of hypertension. Chin J Hypertens. 2011;39:579-615. 
28. Hung HC, Willett W, Merchant A, Rosner BA, Ascherio A, Joshipura KJ. Oral health and peripheral arterial disease. Circulation. 2003;107:1152-7.

29. Yano Y, Stamler J, Garside DB, Daviglus ML, Franklin SS, Carnethon MR, et al. Isolated systolic hypertension in young and middle-aged adults and 31-year risk for cardiovascular mortality: the Chicago heart association detection project in industry study. J Am Coll Cardiol. 2015;65:327-35.

30. Burt BA, Eklund SA. Dentistry, dental practice, and the community. 5ht ed: W.B. Saunders Company; 1992.

31. Darnaud C, Thomas F, Pannier B, Danchin N, Bouchard P. Oral health and blood pressure: the IPC cohort. Am J Hypertens. 2015;28:1257-61.

32. Joshipura K. The relationship between oral conditions and ischemic stroke and peripheral vascular disease. J Am Dent Assoc. 2002;133(Suppl):23S-30S.

33. Ando Y, Ikeda $\mathrm{S}$, Yoshihara A. The reliability of self-assessment of number of remaining teeth using questionnaires. J Oral Health Association. 1997;47: 657-62.

34. Douglass CW, Berlin J, Tennstedt S. The validity of self-reported oral health status in the elderly. J Public Health Dent. 1991;51:220-2.

35. Luo J, Wu B, Zhao Q, Guo Q, Meng H, Yu L, et al. Association between tooth loss and cognitive function among 3063 Chinese older adults: a community-based study. PLoS One. 2015;10:e0120986.

36. Wiener RC, Shen C, Findley PA, Sambamoorthi U, Tan X. The association between diabetes mellitus, sugar-sweetened beverages, and tooth loss in adults: evidence from 18 states. J Am Dent Assoc. 2017;148(7):500.

37. Nascimento GG, Leite FR, Conceição DA, Ferrúa CP, Singh A, Demarco FF. Is there a relationship between obesity and tooth loss and edentulism? A systematic review and meta-analysis. Obes Rev. 2016;17:587-98.

38. Martin H, Poul Erik P, John C, Newell J. Global goals for oral health 2020. Int Dent J. 2011:53:285-8.

\section{Publisher's Note}

Springer Nature remains neutral with regard to jurisdictional claims in published maps and institutional affiliations.

Ready to submit your research? Choose BMC and benefit from:

- fast, convenient online submission

- thorough peer review by experienced researchers in your field

- rapid publication on acceptance

- support for research data, including large and complex data types

- gold Open Access which fosters wider collaboration and increased citations

- maximum visibility for your research: over $100 \mathrm{M}$ website views per year

At $\mathrm{BMC}$, research is always in progress.

Learn more biomedcentral.com/submissions 